\title{
Bactériocines de bactéries lactiques : données récentes sur leur structure, leur mode d'action et leurs déterminants génétiques
}

\author{
Y Cenatiempo 1, JM Berjeaud 1, F Biet ${ }^{1}$, C Fremaux 1,2, \\ Y Hechard ${ }^{1}$, D Robichon ${ }^{1}$ \\ 1 Institut de biologie moléculaire et d'ingénierie génétique, URA CNRS 1172, université de Poitiers, \\ 40, av du Recteur-Pineau, 86022 Poitiers cedex, France; \\ 2 TEXEL, groupe Rhône-Poulenc, Zl de Buxières, BP 10, 86220 Dange-Saint-Romain, France
}

\begin{abstract}
Résumé - Les bactériocines de bactéries lactiques sont l'objet d'une attention toute particulière depuis une dizaine d'années en raison de l'intérêt tant fondamental qu'appliqué qu'elles suscitent. Produites par de nombreuses espèces et dirigées contre des espèces homologues ou plus éloignées, comme Listeria monocytogenes, leur structure primaire a permis de définir une classification en trois classes (lantibiotiques, petites bactériocines non lantibiotiques et bactériocines de grande taille). Leur mode d'action a été également analysé et différents modèles existent pour expliquer la perforation des membranes des cellules cibles de ces bactériocines. La formation de pores membranaires se fait via un récepteur protéique (non lantibiotiques) ou par une insertion directe dans les bicouches phospholipidiques (lantibiotiques), plusieurs molécules d'une même bactériocine agissant en synergie dans tous les cas. L'analyse des déterminants génétiques des bactériocines montre une situation relativement simple dans le cas de la divergicine A (utilisation de la voie générale d'exportation) et plus complexe dans tous les autres cas décrits (voies d'exportation spécifiques faisant appel à des transporteurs de type $\mathrm{ABC}$ ). La compréhension des mécanismes de production, d'immunité, de régulation, mais aussi du mode d'action des bactériocines de bactéries lactiques et des résistances en émergence demande à être affinée à l'échelle moléculaire avant de pouvoir envisager de réelles applications dans les produits laitiers et carnés.
\end{abstract}

bactériocine / lantibiotique / immunité / peptide anti-Listeria

Summary - Bacteriocins of lactic acid bacteria: recent data on their structure, their mode of action and their genetic determinants. Bacteriocins of lactic acid bacteria ( $L A B)$ have been subjected to numerous studies starting 10 years ago because they are of major interest at both basic and applied levels. Bacteriocins are produced by numerous species of $L A B$ and directed towards homologous or eventually heterologous (Listeria monocytogenes) strains. A classification has been proposed in accordance to their primary structures. Three classes have been defined, namely lantibiotics, small non lantibiotics and large size bacteriocins. Their mode of action has been analyzed. The current models are based on pore formation in the membranes of the target cells either by a direct insertion in the phospholipid bilayer (lantibiotics) or after an interaction with a putative protein receptor. In both cases, several bacteriocin molecules acting concomitantly are thought to be involved in pore formation. Genetic 
determinants for bacteriocin synthesis, production and immunity are present in more or less complex gene clusters. The simplest system leads to the synthesis of divergicin A (sec-dependent export) whereas other systems require dedicated export machineries (sec-independent export via ABC transporters). A better knowledge at the molecular level of the basic mechanisms for bacteriocin immunity, production and its regulation, and of the mode of action as well as emerging resistance is still necessary before envisaging extensive technological applications in milk or meat products.

\section{bacteriocin / lantibiotic / immunity / anti-listerial peptide}

\section{INTRODUCTION}

Les bactéries lactiques sont connues pour leur aptitude à produire des composés antibactériens leur permettant de se développer préférentiellement dans divers écosystèmes. Parmi les substances synthétisées, des peptides, dénommés bactériocines, sont produits puis exportés à l'extérieur des cellules productrices. Ils présentent un spectre d'activité plus ou moins large, quelquefois limité aux espèces proches phylogénétiquement des bactéries productrices. Ces bactériocines font l'objet depuis une dizaine d'années d'un nombre croissant d'études, ainsi qu'en témoigne l'abondante littérature scientifique s'y rapportant (voir la dernière revue en date par Jack et al, 1995). En arrière plan de ces nombreux travaux figurent diverses applications potentielles dans les produits laitiers, carnés et marins, la faisabilité de ces applications restant le plus souvent à démontrer. Seuls des acquis fondamentaux seront abordés dans cette courte revue, qui fait un point rapide sur les connaissances actuelles et évoque les pistes sur lesquelles sont lancés les divers laboratoires impliqués dans le domaine.

\section{UNE DÉFINITION SIMPLE}

La définition d'une bactériocine a évolué considérablement pour finalement se simplifier. On considère à présent qu'entre dans cette catégorie toute molécule de nature protéique (fut-ce partiellement), synthétisée par la voie ribosomique et douée d'une activité bactéricide ou bactériostatique. Parmi leurs caractéristiques communes, on peut noter que ces molécules sont synthétisées au sein de la cellule sous la forme d'un précurseur et subissent un processus de maturation au cours de leur export vers le milieu extracellulaire.

\section{LES BACTÉRIES LACTIQUES PRODUISENT DES BACTÉRIOCINES VARIÉES...}

Chez les bactéries lactiques, tous les genres comportent des souches productrices de bactériocines. Si jusque vers la fin des années 1980 , seuls les lactobacilles, les lactocoques et les pédiocoques avaient révélé la présence de bactériocines (voir la revue de Klaenhammer, 1988), ces dernières années ont vu la découverte de nombreuses souches de Leuconostoc et de carnobactéries présentant des activités antagonistes (pour revue, voir Cenatiempo et al, 1995). Si le nombre de bactériocines connues n'a cessé de croître, le nombre réel de structures nouvelles semble stagner dans la mesure où plusieurs laboratoires disséminés dans le monde ont récemment caractérisé des bactériocines identiques ou différant très peu entre elles (voir par exemple Hastings et al, 1991 ; Héchard et al, 1992 ; Felix et al, 1994). On peut considérer que la palette de bactériocines décrites à ce jour correspond à des 
molécules largement optimisées et sélectionnées au cours de l'évolution. Cependant, les expérimentateurs introduisent systématiquement un biais initial en basant leur recherche sur un nombre limité de bactéries indicatrices, souvent une espèce pathogène comme Listeria monocytogenes, et de ce fait aboutissent à une bactériocine déjà identifiée par ailleurs. De plus, une telle approche occulte la présence éventuelle d'une ou plusieurs autres bactériocines synthétisées par la même bactérie, comme la démonstration en a été faite à plusieurs reprises chez les carnobactéries (Worobo et al, 1994 ; Quadri et al, 1994).

\section{... RASSEMBLÉES EN CLASSES ET SOUS-CLASSES}

La classification actuelle est fondée sur la taille et le fait que les bactériocines font l'objet ou non de modifications post-traductionnelles (classification proposée par Klaenhammer en 1993, et révisée à la suite du 1er symposium mondial sur les bactériocines de bactéries lactiques, tenu en avril 1995 à Banff, Canada).

La classe I comporte des petites bactériocines, les lantibiotiques, trouvées non seulement chez les bactéries lactiques mais chez de nombreuses autres bactéries à Gram positif. Ces bactériocines comportent un nombre variable d'acides aminés modifiés (déhydroalanine, déhydrobutyrine, lanthionines, $\beta$-méthyllanthionines) liés par des ponts soufre intrachaînes. Les prototypes sont les nisines $A$ et $Z$ produites par Lactococcus lactis ou encore la lacticine 481 (pour revue, voir différents articles dans l'ouvrage général de De Vuyst et Vandamme, 1994), membres de la sous-classe A, qui rassemble les lantibiotiques cationiques, amphiphiles de forme allongée en hélice agissant sur les cellules cibles en créant des pores membranaires. La nisine A ( 34 acides aminés) est la plus anciennement connue des bactériocines de bactéries lactiques et bénéficie d'un statut particulier du fait de son utilisation en agro-alimentaire dans de nombreux pays. Néanmoins, son spectre d'activité relativement large, touchant à la fois à des espèces pathogènes mais aussi à des bactéries lactiques elles-mêmes, fait qu'elle ne saurait représenter la panacée universelle en terme d'applications. Les nisines $A$ et $Z$, qui ne different entre elles que par un seul acide aminé en position 27 (respectivement une histidine et une asparagine), ont fait l'objet d'analyses par résonance magnétique nucléaire (RMN) et de toute une série de travaux d'ingénierie, à savoir la mutagenèse de divers résidus et les conséquences de ces modifications sur la structure et la fonction de ces lantibiotiques. Il apparaît que les cycles confèrent une certaine rigidité aux divers variants de nisine en solution et qu'ils jouent un rôle indispensable à la fonction bactériocine. II est également acquis que des changements de conformation se produisent au contact des membranes des bactéries cibles de la nisine.

La classe II comprend une plus grande variété de structures, ce qui a nécessité la création de plusieurs sous-groupes. Les bactériocines de la sous-classe lla possèdent en commun d'une part, d'être des petites bactériocines (masse moléculaire inférieure à $10 \mathrm{kDa}$ ) et d'autre part, de posséder une activité anti-Listeria à l'instar de la pédiocine PA-1 (ou AcH) (Gonzalez et Kunka, 1987 ; Bhunia et al, 1988), la plus anciennement connue. L'accumulation de données de séquences a rapidement mis en évidence un motif structural commun à toutes ces bactériocines (sakacines, leucocines, mésentéricine, bavaricine, carnobactériocines, acidocine). II s'agit de l'enchaînement d'acides aminés suivant : YGNGVXC (où $X$ est un acide aminé variable), localisé au voisinage de l'extrémité $\mathrm{NH}_{2}$. II n'est pas encore clairement établi que ce motif joue un rôle direct dans 
l'interaction avec l'enveloppe de Listeria et la mort de cette dernière ou s'il s'agit seulement d'un signal de reconnaissance de la cellule cible. Des travaux récents (Sailer et al, 1995) ont apporté les premiers éléments de structure spatiale d'une de ces bactériocines, la leucocine A ( 37 acides aminés). Parmi les informations apportées par le dichroïsme circulaire et la RMN, ressortent l'absence de structuration en solution aqueuse avec en corollaire la nécessité d'un environnement hydrophobe pour observer une structuration marquée par la présence d'une hélice $\alpha$ amphiphile de la position 19 à 31 , les deux extrémités, en particulier la région comportant la séquence dite anti-Listeria (voir ci-dessus) étant plutôt flexibles.

II semble évident que le nombre de bactériocines anti-Listeria n'est pas infini, ainsi qu'en témoigne le fait que de nombreuses structures découvertes récemment sont très proches voire identiques (par exemple, les identités entre la sakacine $A$ et la curvacine $A$, ou entre les diverses leucocines elles-mêmes très voisines de la mésentéricine Y105). Si la plupart des bactériocines du type Ila sont structuralement apparentées, il n'en demeure pas moins que la pédiocine se démarque notablement des autres membres de la famille au niveau de la région C-terminale, avec la présence d'un pont disulfure du côté C-terminal. L'on pourrait peut-être voir à l'avenir quelques autres exemples de bactériocines proches de la pédiocine, ce qui aurait pour conséquence une dichotomie au sein de la sousclasse Ila.

Les bactériocines de la sous-classe Ilb ont pour trait commun la nécessité de la mise en commun de plusieurs petits peptides aboutissant à un ensemble biologiquement actif. Entrent dans cette catégorie diverses lactococcines, plantaricines ou encore la lactacine F (Allison et al, 1994). Leur spectre d'activité comprend principalement des bactéries lactiques elles-mêmes.
Pour leur part, les bactériocines de la sousclasse Ilc, moins étudiées que les précédentes, comprennent les bactériocines activées par les thiols, comme la lactococcine $B$, tandis qu'entrent dans la sous-classe IId toutes les bactériocines non apparentées aux précédentes. C'est dans cette dernière catégorie que pourrait être intégrée la divergicine A (Worobo et al, 1995) produite par Carnobacterium divergens et qui se démarque par un processus d'export très différent de ceux connus pour les autres bactériocines de bactéries lactiques (voir plus loin).

La classe III a été créée pour rassembler les bactériocines de taille supérieure à $10 \mathrm{kDa}$. À ce jour, seule l'helvéticine J (Jorger et Klaenhammer, 1986), bactériocine de $37 \mathrm{kDa}$ produite par Lactobacillus helveticus a fait l'objet d'études poussées, ce qui tend à indiquer que cette catégorie de bactériocines est peu représentée chez les bactéries lactiques.

En complément à ces trois classes bien définies, une quatrième classe hypothétique (aujourd'hui non reconnue véritablement) pourrait rassembler des bactériocines plus complexes, dont l'existence réelle demande à être établie.

\section{LES BACTÉRIOCINES SONT EXPORTÉES DANS LE MILIEU EXTRACELLULAIRE PAR DES SYSTĖMES SPÉCIFIQUES OU NON}

Les bactériocines se retrouvent dans le milieu extra-cellulaire via un mécanisme mettant en jeu des molécules spécifiques (voies sec-indépendantes), la seule exception notable concerne la divergicine $A$, qui est sécrétée par la voie générale d'exportation (voie sec-dépendante). Les analyses des déterminants génétiques des opérons "bactériocines» montrent généralement la présence d'un gène de transporteur de type 
ABC (pour revue voir Fath et Kolter, 1993) associè à un gène codant une protéine accessoire. Si des motifs structuraux communs sont retrouvés notamment dans les régions participant à l'hydrolyse de l'ATP (motifs $A$ et $B$ ), chaque transporteur de bactériocine semble posséder des séquences qui lui sont propres. En revanche, l'organisation générale de ces transporteurs est fortement conservée avec six segments transmembranaires, la localisation intracellulaire des régions $\mathrm{C}$ - et $\mathrm{N}$-terminales responsables respectivement de l'hydrolyse de l'ATP et, dans le cas de la plupart des bactériocines non lantibiotiques mais aussi vraisemblablement de quelques lantibiotiques, de la maturation de la pré-bactériocine (protéolyse spécifique éliminant la région "leader " $\mathrm{N}$-terminale), ce qui a été démontré pour la pédiocine PA-1 et la lactococcine G (Havarstein et al, 1995 ; Kok, 1995). Ces protéases reconnaissent des sites de clivage différents de ceux habituellement reconnus par les signal-peptidases. Ils sont caractérisés par divers éléments consensus, mais surtout par la présence d'un doublet de glycines, qui précèdent immédiatement la coupure (Havarstein et al, 1994).

Dans l'optique de pouvoir transférer les propriétés antibactériennes des souches productrices de bactériocines vers d'autres souches de bactéries lactiques présentant des potentialités industrielles, la découverte de systèmes d'export et de maturation spécifiques à chaque bactériocine implique leur transfert concomitant et de ce fait complique, a priori, le processus. L'espoir le plus grand réside sans aucun doute dans l'adaptation du système «divergicine $A$ " à d'autres bactériocines. En effet, ayant affaire à une séquence signal classique, il suffirait de greffer cette dernière sur n'importe quelle bactériocine et ce, quelle que soit la cellule hôte, pour obtenir une production extra-cellulaire. Les premiers travaux dans ce sens semblent plus que prometteurs (Worobo, communication personnelle).

\section{LES BACTÉRIOCINES AGISSENT EN PERFORANT L'ENVELOPPE DES CELLULES CIBLES}

Toutes les bactériocines de bactéries lactiques, dont le mode d'action a été étudié, paraissent agir de façon similaire en formant des pores dans la membrane plasmique des cellules cibles. Cependant, sans qu'une explication puisse être fournie, un effet bactériostatique a été décrit pour certaines bactériocines alors que pour d'autres, quelquefois très proches des précédentes, il est clairement bactéricide (voir les exemples de la leucocine UAL-196 et de la mésentéricine $Y 105$ décrits respectivement par Hastings et al, 1991 et Héchard et al, 1992). D'autres différences résident dans la nécessité d'un potentiel membranaire et une action directe sur les structures phospholipidiques (le cas des lantibiotiques, voir l'ouvrage de Jung et Sahl, 1991) ou à l'inverse un mécanisme dit voltage-indépendant, mais nécessitant une interaction initiale avec un récepteur de nature protéique (exemple, la lactococcine $A$ selon les travaux de Van Belkum et al, 1991 et Venema et al, 1995). Dans tous les cas de figure, la formation de pores membranaires a pour conséquence directe l'efflux d'ions ou encore de petites molécules comme des acides aminés (Maftah et al, 1993). La taille des bactériocines de classe I ou II ne permettant pas à une seule molécule de perforer la membrane plasmique, les modèles existants font appel à une action coopérative de plusieurs molécules de bactériocines pour aboutir à la création de pores, dont la taille dépend du nombre de molécules associées. Ces modèles semblent cohérents avec les données expérimentales actuelles, mais demandent à être affinès à l'échelle moléculaire. 


\section{LES BACTÉRIOCINES N'AFFECTENT PAS LES SOUCHES PRODUCTRICES}

Les bactéries productrices pouvant être sensibles à leur propre bactériocine, elles se prémunissent à l'aide d'une protéine qualifiée "d'immunité" (pour revue, voir Abee, 1995). Le seul modèle élaboré pour les bactéries lactiques a été très récemment proposé par Venema et al (1995). La protéine d'immunité, une protéine possédant un large domaine transmembranaire pourrait interagir avec le récepteur potentiel de la bactériocine et empêcherait ainsi l'insertion de cette dernière dans la membrane. Alternativement ou de façon complémentaire, la protéine d'immunité pourrait également interagir directement avec la bactériocine. Le schéma actuel paraît trop simple pour être complètement vrai et généralisable. II va se trouver sans doute modifié le jour où des interactions moléculaires seront établies. Déjà, certains faits troublants ont été rapportés à propos de la localisation cellulaire de la protéine d'immunité. Celle-ci a été trouvée de façon largement majoritaire dans le cytoplasme (Quadri et al, 1995), ce qui non seulement remet en cause le modèle évoqué ci-dessus mais ne permet plus de comprendre facilement le rôle exact de cette protéine.

\section{ORGANISATION DES DÉTERMINANTS GÉNÉTIQUES QUI VA DU TRÈS SIMPLE AU TRĖS COMPLEXE}

L'ensemble des déterminants génétiques responsables de la production d'une bactériocine sont en général trouvés au voisinage les uns des autres groupés en une ou plusieurs unités transcriptionnelles. Elles sont portées par des éléments génétiques mobiles (transposons, plasmides) et exceptionnellement localisées sur le chromosome bactérien. La figure 1 montre quelques exemples caractéristiques allant d'une situa- tion simple (deux gènes pour la divergicine A) à plus compliquée (11 gènes dans le cas de la nisine), en passant par des situations intermédiaires (quatre ou cinq gènes dans le cas respectif de la lactococcine $A$ et de la mésentéricine Y105). Des situations encore plus complexes sont également en cours de décryptage (par exemple, les opérons plantaricine A analysés par Diep et al, 1995, rassemblés sur une longueur de $20 \mathrm{kpb}$ ). Hormis les diverses fonctions mentionnées tout au long de cette revue, auxquelles sont associés divers gènes mis en évidence, l'analyse génétique a apporté des informations supplémentaires. II apparaît par exemple que la synthèse de certaines bactériocines fait l'objet d'une régulation par le biais de systèmes à deux composants (histidine kinase activée par un signal externe, régulateur actif après phosphorylation), la bactériocine pouvant être par ailleurs un inducteur non exclusif du processus de synthèse. De plus, dans le cas de la nisine, plusieurs gènes, donc plusieurs protéines, apparaissent impliqués dans le phénomène d'immunité. L'on ne sait pas par ailleurs si d'autres gènes chromosomiques ou portés par d'autres éléments génétiques mobiles ne jouent pas un rôle essentiel dans ce qui paraissait voici quelques années une histoire simple, mais qui s'est compliquée à souhait avec l'accumulation de donnees nouvelles.

\section{QUE FAIRE AUJOURD'HUI ET QUELLES RECHERCHES PRIVILÉGIER ?}

Les pistes ne manquent pas, même pour les plus malchanceux, qui viennent de trouver une bactériocine identique à l'une ou l'autre déjà connue. En effet, rien ne prouve que l'ensemble des déterminants génétiques sera identique et que la souche productrice nouvellement identifiée ne possède pas des avantages sur ses glorieuses 
A

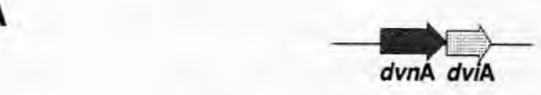

B

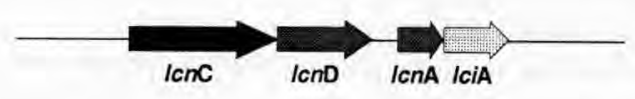

C

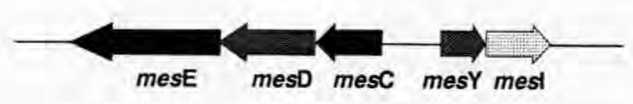

D

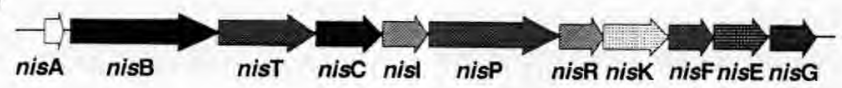

Fig 1. Représentation schématique des opérons divergicine $A(A)$, lactococcine $A(B)$, mésentéricine Y105 (C) et nisine A (D). Les flèches indiquent l'orientation des gènes, qui sont organisés en une seule ou plusieurs unités transcriptionnelles convergentes ou divergentes. Les gènes de structure des précurseurs de bactériocines sont respectivement dénotés $d v n A$, IcnA, mesY et nisA. Signification et rôle (avéré ou potentiel) des autres gènes : dviA, gène de la protéine d'immunité vis-à-vis de la divergicine; $I c n C$ et $l c n D$, gènes impliqués dans l'export de la lactococcine $\mathrm{A}$ (codant respectivement un $\mathrm{ABC}$ transporteur et un facteur accessoire), IciA, gène codant une protéine d'immunité ; mesE et mes $\mathrm{D}$, gènes impliqués dans l'export de la mésentéricine $\mathrm{Y} 105$, mesC, phase ouverte de lecture correspondant à un gène codant une protéine non identifiée, mesl, gène codant une protéine d'immunité ; nis $\mathrm{B}$, gène codant une protéine responsable de la maturation post-traductionnelle de la nisine (réactions de déshydratation d'acides aminés), nisT et nisC, gènes codant les protéines de transport de la nisine (transporteur $\mathrm{ABC}$ et facteur accessoire), nisl, nisF, nisE, nisG, gènes codant un ensemble de protéines participant à limmunité vis-à-vis de la nisine (les produits de nisF et nisE constituant également un système transporteur de type $A B C$ ) ; nisP, gène d'une protéase à sérine de type subtilisine clivant le précurseur de la nisine ; nisR et nisK, système de régulation à deux composants. Schémas adaptés de Stoddard et al, 1992 ; Fremaux et al, 1995 ; Siegers et Entian, 1995 ; Worobo et al, 1995.

Schematic representation of the divergicin $A(A)$, lactococcin $A(B)$, mesentericin $Y 105(C)$ and nisin $A$ (D) operons. The arrows indicate the orientation of the genes, which are organized as single-or polycistronic tandem array or diverging units. The structural genes for bacteriocin precursors are respectively denoted $\operatorname{dvn} A$, Icn $A$, mes $Y$ and nis $A$. Significance and role (shown or putative) of other genes: $\mathrm{dvi} A$, divergicin immunity gene; Icn $C$ and $\operatorname{lcn} D$, lactococcin $A$ export genes (encoding respectively an $A B C$ transporter and an accessory factor), Ici $A$, immunity gene; mes $E$ and mes $D$, mesentericin $Y 105$ export genes, mes $C$, open reading frame encoding an unidentified protein, mesl, immunity gene; nis $B$, gene involved in the post-translational maturation of nisin (dehydration of amino acids), nis $T$ and nis $C$, nisin export genes ( $A B C$ transporter and accessory factor), nis $I$, nis $F$, nis $E$ and nis $G$, genes encoding proteins involved in imunity to nisin (the gene products of nis $F$ and nis $E$ also constitute an $A B C$ transport system); nis $P$, gene for a serine-protease (subtilisin type) responsible for the cleavage of the nisin precursor; nis $R$ and nis $K$, genes for a two component regulatory system. Adapted from Stoddard et al, 1992; Fremaux et al, 1995; Siegers and Entian, 1995; Worobo et al, 1995. 
aînées (capacités de production de bactériocine et de développement supérieurs dans des milieux complexes notamment). Cependant, les principaux développements passent par l'identification de tous les partenaires impliqués dans la synthèse de chaque bactériocine et sa régulation, le transport, l'immunité, le mode d'action sur la cellule cible. D'autre part, la mise en évidence, par toutes les équipes travaillant dans le domaine, de mutants de souches cibles comme Listeria monocytogenes, devenues résistantes aux bactériocines implique que l'on s'intéresse à ce phénomène, qui n'est pas sans rappeler ce qui est classiquement observé avec les antibiotiques. II semble d'ores et déjà que l'on puisse s'orienter dans deux directions distinctes, l'une s'attachant à comprendre un phénomène adaptatif des cellules cibles (modification de l'enveloppe externe), l'autre recherchant des gènes mutés, ces derniers pouvant fournir une clé à la compréhension du mode d'action.

D'autres approches doivent permettre d'améliorer des outils encore peu performants ou disponibles en quantités insuffisantes (vecteurs navettes, bactériocines naturelles, synthétiques ou recombinantes par exemple), afin d'accroître nos connaissances fondamentales et d'aller vers des applications, limitées pour le moment à la fois par des problèmes techniques mais aussi réglementaires, tenant notamment à l'utilisation d'organismes génétiquement modifiés.

\section{RÉFÉRENCES}

Abee T (1995) Pore-forming bacteriocins of Gram-positive bacteria and self-protection mechanisms of producers organisms. FEMS Microbiol Lett 129, 1-10

Allison GE, Fremaux C, Klaenhammer TR (1994) Expansion of bacteriocin activity and host range upon complementation of two peptides encoded within the lactacin F operon. J Bacteriol 176, 2235-2241

Bhunia AK, Johnson MC, Ray B (1988) Purification, characterization and antimicrobial spectrum of a bacte- riocin produced by Pediococcus acidilactici. J Appl Bacteriol 65, 261-268

Cenatiempo Y, Biet F, Fremaux C, Héchard Y, Robichon D (1995) What's new in bacteriocins of Carnobacterium, Leuconostoc and other lactics? Can $J$ Microbiol (sous presse)

De Vuyst L, Vandamme EJ (1994) Bacteriocins of lactic acid bacteria. Blackie Academic and Professional, London

Diep DB, Havarstein LS, Nes I (1995) A bacteriocin substance functions as inducing signal for bacteriocin synthesis in Lactobacillus plantarum $\mathrm{C} 11$. Abstract P15, Bacteriocins of lactic acid bacteria - Applications and fundamentals, Banff, Alberta, Canada

Fath MJ, Kolter R (1993) ABC transporters: bacterial exporters. Microbiol Rev 57, 995-1017

Felix JV, Papathanasopoulos MA, Smith AA, von Holy A, Hastings JW (1994) Characterization of leucocin B-Ta11a: a bacteriocin from Leuconostoc carnosum Ta11a isolated from meat. Curr Microbiol 29, 207-212

Fremaux C, Héchard Y, Cenatiempo Y (1995) Mesentericin Y105 gene clusters in Leuconostoc mesenteroides Y105. Microbiology 141, 1637-1645

Gonzalez CF, Kunka BS (1987) Plasmid-associated bacteriocin production and sucrose fermentation in Pediococcus acidilactici. Appl Environ Microbiol 53. 2534-2358

Hastings JW, Sailers M, Johnson K, Roy KL, Vederas JC, Stiles ME (1991) Characterization of leucocin A-UAL187 and cloning of the bacteriocin gene from Leuconostoc gelidum. J Bacteriol 173, 7491-7500

Havarstein LS, Holo $H_{4}$ Nes I (1994) The leader peptide of colicin V shares consensus sequences with leader peptides that are common among peptide bacteriocins produced by Gram-positive bacteria. Microbiology 140, 2383-2389

Havarstein LS, Diep DB, Nes I (1995) A family of bacteriocin $A B C$ transporters carry out proteolytic processing of their substrates concomitant with export. Mol Microbiol 16, 229-240

Héchard Y, Dérijard B, Letellier F, Cenatiempo $Y$ (1992) Characterization and purification of mesentericin Y105, an anti-Listeria bacteriocin from Leuconostoc. mesenteroides. J Gen Microbiol 138, 2725-2731

Jack RW, Tagg J, Ray B (1995) Bacteriocins of grampositive bacteria. Microbiol Rev 59, 171-200

Joerger MC, Klaenhammer TR (1986) Characterization and purification of helveticin $\mathrm{J}$ and evidence for a chromosomally determined bacteriocin produced by Lactobacillus helveticus 481. J Bacteriol 167, 439-446

Jung G, Sahl H-G (1991) Nisin and novel lantibiotics. ESCOM, Leiden

Klaenhammer TR (1988) Bacteriocins of lactic acid bacteria. Biochimie 70, 337-349 
Klaenhammer TR (1993) Genetics of bacteriocins produced by lactic acid bacteria. FEMS Microbiol Rev $12,39-85$

Kok J (1995) Secretion systems for lantibiotics and nonlantibiotics. Abstract 3.2, Bacteriocins of lactic acid bacteria - Applications and fundamentals, Banff, Alberta, Canada

Mafath A, Renault D, Vignoles C, Héchard Y, Bressolier P, Ratinaud MH, Cenatiempo Y, Julien R (1993) Membrane permeabilization of Listeria monocytogenes and mitochondria by the bacteriocin mesentericin Y105. J Bacteriol 175, 3232-3235

Quadri LEN, Sailer M, Roy KL, Vederas JC, Stiles ME (1994) Chemical and genetic organization of bacteriocins produced by Camobacterium piscicola LV17B. $J$ Biol Chem 269, 12204-12211

Quadri LEN, Sailer M, Terebiznek M, Roy KL, Vederas JC, Stiles ME (1995) Characterization of the protein conferring immunity to the antimicrobial peptide carnobacteriocin B2 and expression of carnobacteriocin B2 and BM1. J Bacteriol 177, 1144-1151

Sailer M, Niemczura WP, Nakashima TT, Stiles ME, Vederas JC (1995) 3D structure of leucocin A in environments that mimics membranes. Abstract P23, Bacteriocins of lactic acid bacteria - Applications and fundamentals, Banff, Alberta, Canada
Siegers K, Entian KD (1995) Genes involved in immunity to the lantibiotic nisin produced by Lactococcus lactis 6F3. Appl Environ Microbiol 61, 1082-1089

Stoddard GW, Petzel JP, van Belkum MJ, Kok J, McKay LL (1992) Molecular analyses of the lactococcin A gene cluster from Lactococcus lactis subsp lactis biovar diacetylactis WM4. Appl Environ Microbiol $58,1952-1961$

Van Belkum MJ, Kok J, Venema G, Holo H, Nes I, Konings WN, Abee T (1991) The bacteriocin lactococcin A specifically increases permeability of lactococcal cytoplasmic membranes in a voltage-independent, protein-mediated manner. J Bacteriol 173, 7934-7941

Venema K, Venema G, Kok, J (1995) Lactococcal bacteriocins: mode of action and immunity. Trends Microbiol 3, 299-304

Worobo RW, Henkel T, Sailer M, Roy K, Vederas JC, Stiles ME (1994) Characteristics and genetic determinants of a hydrophobic peptide bacteriocin, carnobacteriocin A, produced by Carnobacterium piscicola LV17A. Microbiology 140, 517-526

Worobo RW, Van Belkum MJ, Sailer M, Roy K, Vederas JC, Stiles, ME (1995) A signal peptide secretiondependent bacteriocin from Carnobacterium divergens. J Bacteriol 177, 3143-3149 